\title{
OPTICAL MEASUREMENTS ON SOLIDS OF POSSIBLE INTERSTELLAR IMPORTANCE
}

\author{
DONALD R. HUFFMAN and JAMES L. STAPP* \\ Physics Dept., University of Arizona, Tucson, Ariz. 85721, U.S.A.
}

\begin{abstract}
Optical constants of olivine $-(\mathrm{Mg}, \mathrm{Fe})_{2} \mathrm{SiO}_{4}$ and magnetite $-\mathrm{Fe}_{3} \mathrm{O}_{4}$ are presented for the wavelength range from near infrared to far ultraviolet. A feature occurs in the optical constants of olivine at about $1460 \AA$, but no structure that could give rise to a $2200 \AA$ interstellar feature is found. The most peculiar characteristic of the magnetite results is the large change of optical properties with temperature in the infrared.
\end{abstract}

\section{Introduction}

Optical constants of solids over wide wavelength ranges needed for Mie scattering calculations relating to interstellar and circumstellar solids have not been readily available in the scientific literature. We have begun a program of measuring optical constants of likely interstellar and circumstellar solids from far infrared to far ultraviolet. Results for two solids, olivine and magnetite, are presented in this paper. These are perhaps the best possibilities from the two groups of solids of interest to us, i.e., silicates and oxides of iron.

The reason for interest in silicates, apparent from some of the papers being presented in this symposium, is the likely identification of silicates in various circumstellar regions by their characteristic absorption bands in the $10 \mu$ region. In addition it has been suggested (Huffman and Stapp, 1971) that enstatite silicate in certain size ranges could produce a $2200 \AA$ band corresponding to the prominent interstellar feature. The extinction 'bump' expected in small particles of enstatite is produced by the onset of strong absorption near $2100 \AA$. Whether this is a general characteristic of silicates has not been known because of the lack of ultraviolet measurements on other solids.

Our interest in iron oxides is for the following reasons: (1) the possibility that some of the diffuse interstellar bands may be due to $\mathrm{Fe}^{+++}$in such solids, and (2) the alignment of grains necessary to produce interstellar polarization may require ferromagnetic solids. The $\mathrm{Fe}^{+++}$-diffuse-band-theory was suggested several years ago by different authors independently (Manning, 1970; Dorschner, 1970; Huffman, 1970). Manning and Dorschner discussed the possibility of iron ions in various silicates. Our calculations and measurements show, however, that the oscillator strength of $\mathrm{Fe}^{+++}$ is generally too low to produce a band of the observed strenght of $\lambda 4430$, unless the bands are due to iron ions in a crystal with cooperative magnetic ordering (ferromagnetism, antiferromagnetism, or ferrimagnetism). In magnetic iron oxides such as $\alpha-\mathrm{Fe}_{2} \mathrm{O}_{3}, \gamma-\mathrm{Fe}_{2} \mathrm{O}_{3}, \mathrm{Fe}_{3} \mathrm{O}_{4}$, and $\mathrm{MgFe}_{2} \mathrm{O}_{4}$, the oscillator strengths of the absorption

* Present address: Kirtland A.F.B., Albuquerque, N.M.

Greenberg and Van de Hulst (eds.), Interstellar Dust and Related Topics, 297-301. All Rights Reserved. Copyright (C) 1973 by the IAU. 
bands can be as much as three orders of magnitude greater than in non magnetic solids because of the internal magnetic interaction coupling adjacent iron atoms (Huffman, 1969, and unpublished results). Furthermore, this cooperative effect of neighboring iron atoms in the solid is just the feature that Purcell and Spitzer (1971) have concluded is necessary for alignment by a Davis-Greenstein-type mechanism in $10^{-6} \mathrm{G}$ interstellar magnetic fields.

\section{Determination of Optical Constants}

For these reasons, we have made measurements to determine optical constants from near infrared to far ultraviolet for magnetite $-\mathrm{Fe}_{3} \mathrm{O}_{4}$, and olivine $-(\mathrm{Mg}, \mathrm{Fe})_{2} \mathrm{SiO}_{4}$. The samples were carefully selected natural specimens that were cut, polished, and etched to provide specularly reflecting surfaces with very small-scale surface roughness. Reflectance measurements were made at near-normal incidence from about $3 \mu$ to about $400 \AA$ in wavelength, and the optical constants determined by a KramersKronig analysis of the reflectance data (Stern, 1963). Figures 1 and 2 show the energy dependence of the real and imaginary parts of the complex optical constant $N$, defined as

$$
N=n+i k .
$$

$n$ is the index of refraction and $k$ is commonly called the extinction coefficient.

Although the combination of specular reflectance measurements and KramersKronig analysis is the most widely used and probably the best method for obtaining

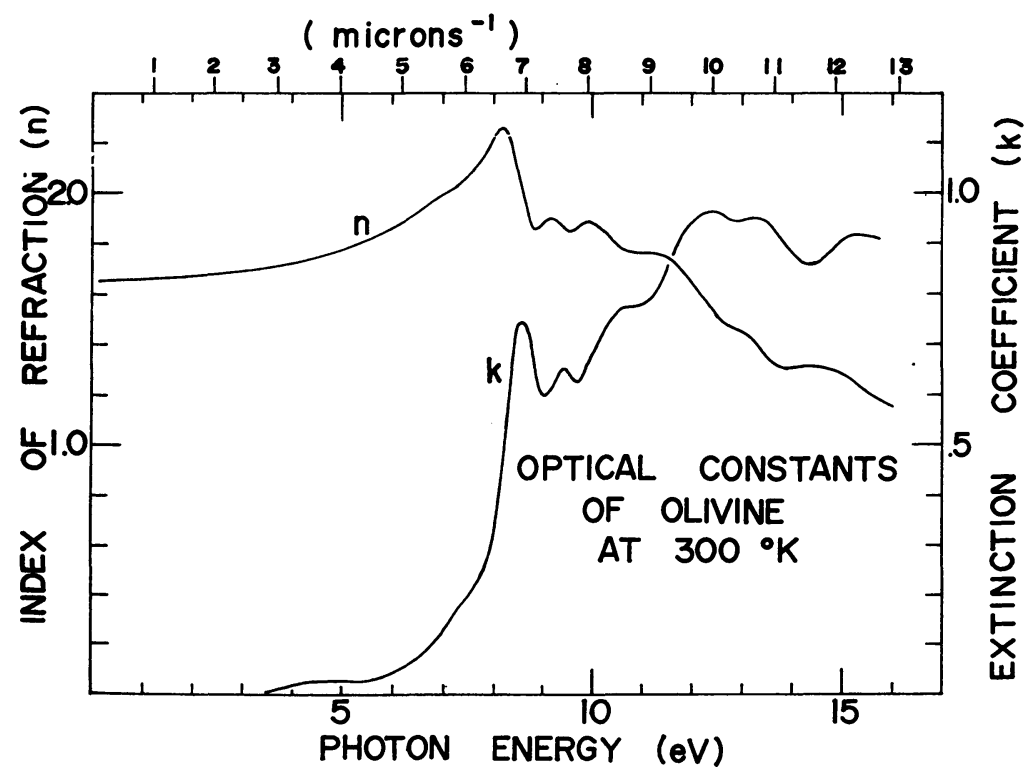

Fig. 1. Optical constants of Olivine $(\mathrm{Mg}, \mathrm{Fe})_{2} \mathrm{SiO}_{4}$ at about $300 \mathrm{~K}$. 


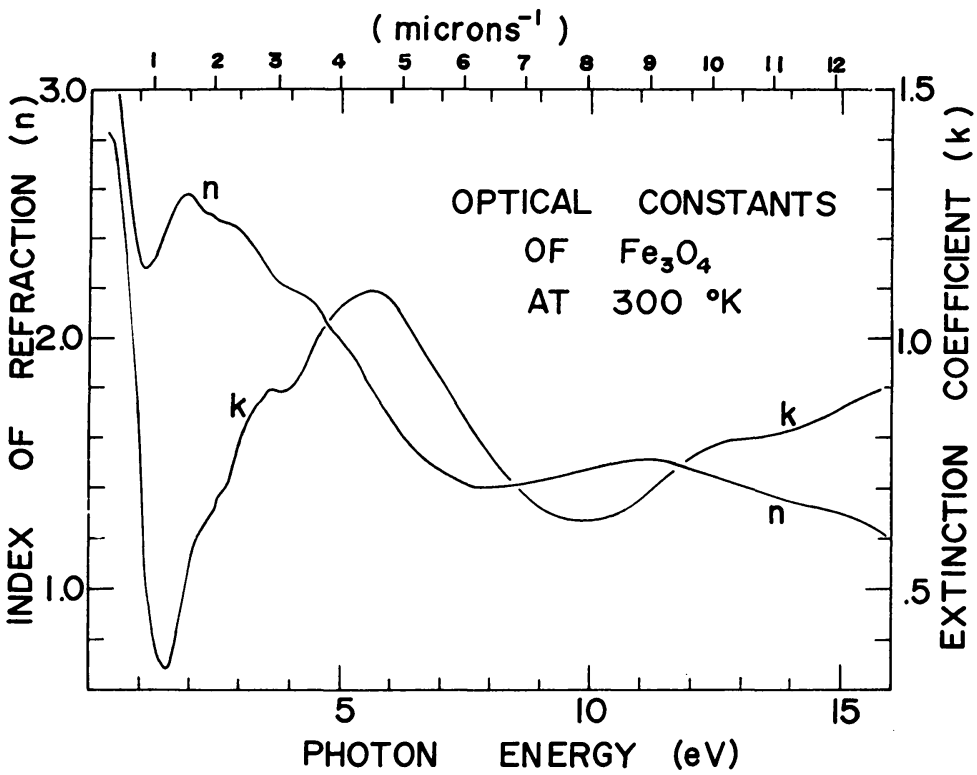

Fig. 2. Optical constants of magnetite $\mathrm{Fe}_{3} \mathrm{O}_{4}$ at about $300 \mathrm{~K}$. Note that the scales for both optical constants do not start at 0 .

optical constants in the highly absorbing ultraviolet regions, various possibilities for error exist. The most difficult error to circumvent may be caused by the extrapolation of the reflectance data to infinite energy required by the Kramers-Kronig integral (Stern, 1963). Although the wide range of data used here (extending to $30 \mathrm{eV}$ ) minimizes this problem, the unknown extrapolation to infinite energy can still cause appreciable error. In addition, surface films, residual surface roughness, and amorphous layers introduced by polishing can cause severe problems in the far ultraviolet. For these reasons we feel that it would be a mistake to trust any determinations of this kind to better than $10 \%$ in highly absorbing regions $(k>0.1)$. A revealing example of the kind of errors involved can be obtained by comparing results on $\mathrm{MgO}$ obtained independently by two well-respected groups of workers in this field (Williams and Arakawa, 1967; Roessler and Walker, 1967). Although MgO can be artificially grown as a very pure single crystal which cleaves easily, leading to atomically smooth and clean surfaces, discrepancies between the two independent measurements of optical constants of 10-20\% are present. Despite possible uncertainties in magnitude, the method is usually quite reproducible in revealing structure in the optical constants curves.

\section{Discussion of Results}

The main value of these results is that they make possible realistic scattering calculations on two new materials. A few comments on the results are perhaps in order. Regarding the olivine, strongly increasing absorption does not set in until about $7 \mathrm{eV}$ 
( $\sim 1800 \AA$ ), in contrast to the onset of absorption near $2100 \AA$ for the silicate enstatite. Thus a feature near $2200 \AA$ is not a general characteristic of silicates, and Mie calculations using these optical constants for olivine will not produce a $2200 \AA$ 'bump' for any sizes. This, of course, is not an argument against such a silicate being a component of the interstellar medium. There is a fairly strong feature in the extinction coefficient of olivine near $8.5 \mathrm{eV}(1460 \AA)$, but there is no obvious structure in the interstellar extinction curve near this wavelength. Measurements were also made at $100 \mathrm{~K}$ on olivine with very little change in the resulting optical constants.

The optical constants of magnetite are very different. There is a pronounced minimum in both $n$ and $k$ in the near infrared when the solid is at $300 \mathrm{~K}$. Toward longer wavelengths there is a rapid rise in both optical constants, and toward shorter wavelengths a lesser increase. The effect of lower temperature is very great below $1 \mathrm{eV}$ and very small above $2 \mathrm{eV}$. This is probably due to the fact that magnetite undergoes a so-called metal-to-semiconductor transition at about $119 \mathrm{~K}$ (Adler, 1968). Above this temperature it behaves somewhat like a metal, and the optical constants below $1 \mathrm{eV}$ show this. At temperatures lower than $119 \mathrm{~K}$, where magnetite behaves like a semiconductor, there is a large decrease in reflectance below $1 \mathrm{eV}$. Although the low temperature studies of magnetite in the infrared have not progressed to where we can determine optical contants below $1 \mathrm{eV}$, preliminary indications are that $k$ will decrease toward 0 and $n$ will level off. These variations of optical properties with temperature are among the largest occurring in common solids, and could have important consequences if magnetite is present in space. There is some fine structure in the visible region in magnetite which is not apparent on this energy scale and at $300 \mathrm{~K}$. This is the structure (due to iron ions in the solid), that might provide explanations for some diffuse interstellar features. Further work is being done at low temperatures to explore these small (on this scale) but potentially significant variations of optical constants.

Further work now in progress at this laboratory will provide optical constants measurements comparable to Figures 1 and 2 for other possible interstellar solids. We also have begun detailed determinations of optical constants for silicates and other solids in the $10 \mu$ and $20 \mu$ regions.

\section{Acknowledgements}

We would like to gratefully acknowledge the collaboration in making the far ultraviolet measurements of $\mathrm{H}$. Fritzsche, J. Freeouf, J. W. Osmun, and K. Murase of the University of Chicago. This research was supported in part by USAF Office of Scientific Research, contract number F44620-70-C-0029.

\section{References}

Adler, D.: 1968, in Solid State Physics, Vol. 21, Academic Press, New York, p. 109.

Dorschner, J.: 1970, Astron. Nachr. 292, 107. 
Huffman, D. R.: 1970, Astrophys. J. 161, 1157.

Huffman, D. R.: 1969, J. Appl. Phys. 40, 1334.

Huffman, D. R. and Stapp, J. L.: 1971, Nature Phys. Sci. 229, 45.

Manning, P. G.: 1970, Nature 226, 829.

Purcell, E. M. and Spitzer, L.: 1971, Astrophys. J. 167, 31.

Roessler, D. M. and Walker, W. C.: 1967, J. Opt. Soc. Am. 57, 835.

Stern, F.: 1963, in Solid State Physics, Vol. 15, Academic Press, New York, p. 300.

Williams, M. W. and Arakawa, E. T.: 1967, J. Appl. Phys. 38, 5272. 